Research Article

\title{
Characteristics Analysis of Generalized Rock Quality Designation (RQD) Based on Degree of Joint Development
}

\author{
Lin Jun $\mathbb{D}^{1},{ }^{1}$ Sha Peng $\mathbb{D}^{2},{ }^{2}$ Gao Shuyu, ${ }^{3}$ and Ni Jiji $\mathbb{D}^{2}$ \\ ${ }^{1}$ Zhejiang Institute of Communications Co., Ltd., Hangzhou, Zhejiang 310000, China \\ ${ }^{2}$ College of Civil Engineering, Shaoxing University, Shaoxing, Zhejiang 312000, China \\ ${ }^{3}$ Key Laboratory of Rock Mechanics and Geohazards of Zhejiang Province, Shaoxing, Zhejiang 312000, China \\ Correspondence should be addressed to Sha Peng; shapeng@usx.edu.cn
}

Received 7 April 2021; Revised 1 June 2021; Accepted 21 July 2021; Published 5 August 2021

Academic Editor: Xin-Jiang Wei

Copyright ( 92021 Lin Jun et al. This is an open access article distributed under the Creative Commons Attribution License, which permits unrestricted use, distribution, and reproduction in any medium, provided the original work is properly cited.

Rock quality designation (RQD) is widely adopted as a fundamental tool in characterizing rock masses since it was devised in 1964. Since the conventional RQD calculation is limited due to its dependence on the selected threshold, previous research introduced generalized RQD to adequately reflect the anisotropy and scale effect of RQD. However, the influence of the joint development inside rock mass on generalized RQD remains unclear. The objective of this work is to investigate characteristics of the generalized RQD in view of different development degrees of discontinuities in rock mass, including spacing (density) and trace length. Three-dimensional fracture network modelling is employed to simulate the actual rock mass of open-pit iron mine in China. Virtual scanlines are set to obtain RQD values in different directions. The results primarily show that the generalized RQD should be introduced to calculate the RQD with different thresholds to fully reflect the anisotropy of rock mass. The optimal threshold can be obtained based on an anisotropic coefficient, which is defined by $\left(R Q D_{\max }-R Q D_{\min }\right)$. It is also indicated that the fracture spacing has a great influence on both the anisotropy of RQD and the selection of the optimal threshold. The optimal threshold of the generalized RQD increases with the increase in the fracture spacing. In addition, the scale effect of RQD in different models is discussed by changing the length of the scanlines. The longer the scanlines we set, the more stable RQD value can be obtained in the model. It is recommended to fit much longer scanline to get realistic RQD in heavily fractured rock mass.

\section{Introduction}

The presence of discontinuities plays an essential role in the strength and stability of rock masses, which controls the damage characteristics of rock mass [1-4]. Rock quality designation (RQD) is an important parameter in evaluating the quality of rock masses using drill core cataloging. This parameter was initially proposed by [5]. It provides a quite simple description of rock mass quality qualitatively [6]. At present, it has been widely employed as the foundational parameter in the rock mass classification system such as Rock Mass Rating (RMR) and the Rock Quality Tunneling Index (Q-system) [7-11]. RQD is internationally adopted in areas of mines, rock mechanics, and engineering geology
$[12,13]$. The value of RQD is expressed as follows [14], where $\sum_{i=1}^{n} x_{i}$ is all pieces of core with length greater than $100 \mathrm{~mm}$ and $X$ is the total length of the drilling borehole.

$$
\mathrm{RQD}=100 \sum_{i=1}^{n} \frac{x_{i}}{X} \times 100 \%
$$

However, three limitations in the aforementioned calculation principles are observed when obtaining RQD. First, the "threshold value of $10 \mathrm{~cm}$ " is used as the only criterion for judging rock quality. Whether this threshold can be used as the most reasonable threshold value for distinguishing rock mass has been questioned by many scholars [15-19]. Second, due to the geological survey level and engineering 
cost, the value of RQD can be different for a given location when measured from cores with different drilling orientations $[20,21]$. The conventional RQD cannot fully reflect the heterogeneity and anisotropy of the rock mass [22, 23]. Third, traditional methods often directly calculate the size of RQD based on the length of each section of the core in the borehole, ignoring the scale effect of RQD [24-26].

To overcome the aforementioned limitations, many domestic and foreign scholars have conducted relevant research. The authors in [27] found that RQD changes with the size of the scanline; that is, RQD has scale effect. Thus, the authors proposed corresponding empirical formulas as follows:

$$
\mathrm{RQD}=100(1+\lambda t) e^{-\lambda t}
$$

where $\lambda$ is the density of structural surface (average number of structural surfaces per meter of scanline) and $t$ is the selected threshold for calculation. Harrison believed that a reasonable selection of thresholds can expand the range of RQD change and proposed a corresponding equation [16]. Based on the relationship between threshold and RQD change, the generalized RQD was suggested in light of rock mass anisotropy [28]. The authors in [29] investigated the generalized RQD through in situ cataloging of discontinuities and 2D network simulation technology. The results pointed out the limitations in using $0.1 \mathrm{~m}$ as the only threshold. The authors in [30] conducted the three-dimensional fracturing network method to simulate rock slope of the Baihetan hydropower station. By arranging numerous scanlines, it is indicated that the generalized RQD has obvious spatial and scale effects.

The RQD index is influenced by different parameters which mainly include joint orientation, joint spacing, fracture frequency, and joint roughness [31]. Although predecessors have conducted extensive research on the limitations of the traditional method to calculate the generalized RQD, how the development of the discontinuities in rock mass will affect the characteristics of the generalized RQD remains unclear. Therefore, this study adopts $3 \mathrm{D}$ fracture network simulation $[32,33]$ to generate numerous models of an actual rock mass by considering different jointing degrees. A series of virtual scanlines were arranged in different directions in each model. The influence of joint development on the anisotropy of the generalized RQD is analyzed. The scale effect of generalized RQD is also studied by changing the length of the scanline, which provides a certain theoretical basis for selecting a reasonable drilling depth of rock projects.

\section{Model Establishment of an Actual Rock Mass}

2.1. Study Area and Geological Setting. Practical investigations on RQD measurements were carried out at Anqian open-pit iron mine, which is located in southwest of Anshan, in Liaoning Province, China. The strata of this area were precipitated in medium and high metamorphic series with NW-SE trend. The major rock types at the open-pit slope are ferruginous quartzite, schist, and phyllite of the Anshan Group, which belongs to Precambrian.

We adopted the noncontact sampling method to conduct geological logging at Xudonggou stope using the ShapeMetriX3D system [34]. Four key discontinuity parameters of 258 discontinuities, i.e., orientation, number of sets, set spacing, and mean trace length, were collected and analyzed. Three sets of discontinuities intersect the rock mass of the stope: a bedding and two major joints, according to the variation range of dip angle (see Figure 1).

2.2. Model Parameters. Gu [35] divided the joints of rock mass into five levels according to the scale of structures: I, II, III, IV, and V. Among them, the IV-grade structural planes extend from tens of centimeters to $20-30 \mathrm{~m}$, with a large number distributed randomly, thereby affecting the integrity and mechanical properties of the rock mass. This issue is the subject of statistical analysis and simulation of structural planes. Studies have found that when the number of structural planes is constant, the development degree of discontinuities is mainly related to their spacing and ductility, and the occurrence and distribution type of structural planes have a minimal, even negligible, effect on it [36].

Regarding the quantitative description of structural plane spacing and ductility, the International Society for Rock Mechanics [37] proposed the ductility of structural planes into five levels $(<1,1-3,3-10,10-20$, and $>20 ; \mathrm{m})$ and the spacing into 7 levels $(<20,20-60,60-200,200-600$, $600-2000,2000-6000$, and $>6000 ; \mathrm{mm})$. Based on the preceding classification and the scale of the case study, this work only selects seven representative structural plane spacing values and five representative structural ductility ranges, which are tabulated in Table 1.

The discontinuities inside the rock mass are mainly divided into occurrence, ductility, and density (spacing). The accuracy of the above elements greatly determines the accuracy of the 3D joint network model [38]. Three sets of discontinuities at Xudonggou stope are developed in the rock mass. The distribution of occurrence follows the Fisher distribution [39], which contains only one parameter, the Fisher constant $K$. The spacing and trace length distribution obey the negative exponential distribution [40]. The specific parameters of 3D fracture network simulation are listed in Table 2. According to the result of [41], the value of CP3 is 2.0 for a uniform distribution of fracture orientation. For most fracture geometries, CP3 will vary between 1.0 and 3.0. Therefore, the value of CP3 is set to 2.0 here. Meanwhile, the density of the discontinuities can be calculated from the spacing presented in Table 1 . The solution method is based on the work of [42]; and the calculation process is not mentioned in this study.

2.3. Model Generation and Scanline Layout. The existence of discontinuities causes the rock mass to have an obvious scale effect. The parameters of rock mass change with the sampling scale [43]. With the increase in the sample to a certain critical value, the parameters of rock mass tend to be stable. The sampling size corresponding to the critical value is the 


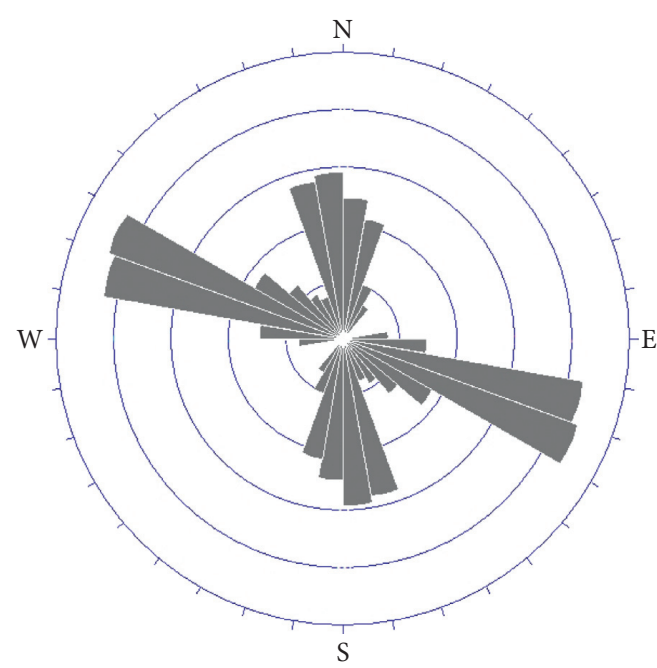

(a)

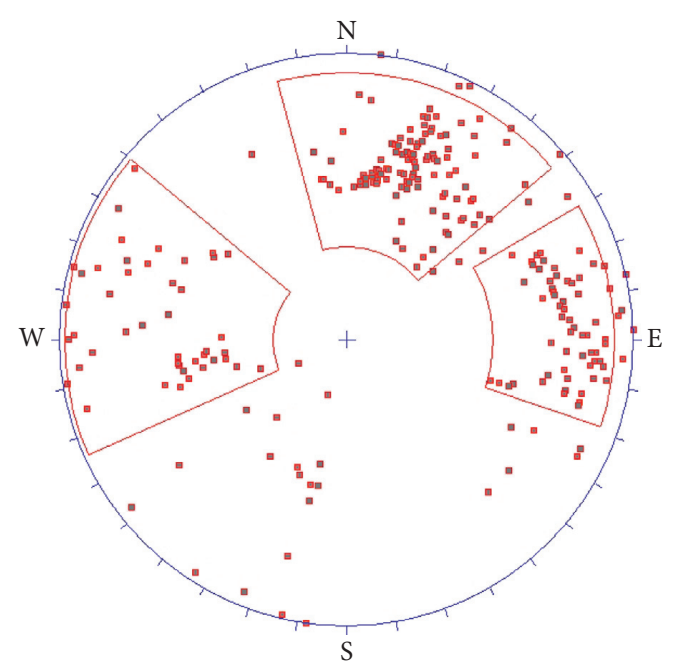

(b)

Figure 1: Joints distribution at Anqian open-pit iron mine: (a) rose diagram of joint and (b) pole distribution.

TABLE 1: Representative values of joint spacing and persistence.

\begin{tabular}{|c|c|c|c|c|c|c|c|}
\hline Scalability (unit: $\mathrm{m}$ ) & Extremely low & Low & Medium & High & Extremely high & & \\
\hline & $1-3$ & $3-8$ & $8-14$ & $14-20$ & $20-30$ & & \\
\hline Spacing (unit: $\mathrm{m}$ ) & $\begin{array}{c}\text { Extremely dense } \\
0.2\end{array}$ & $\begin{array}{c}\text { Very dense } \\
0.5\end{array}$ & $\begin{array}{c}\text { Dense } \\
0.8\end{array}$ & $\begin{array}{c}\text { Medium } \\
1\end{array}$ & $\begin{array}{c}\text { Wide } \\
1.5\end{array}$ & $\begin{array}{c}\text { Very wide } \\
3\end{array}$ & $\begin{array}{c}\text { Extremely wide } \\
6\end{array}$ \\
\hline
\end{tabular}

TABle 2: Parameters of 3D fracture network.

\begin{tabular}{lcccc}
\hline Group & Structure occurrence & & Spacing distribution & Volume density \\
\hline Set 1 & Occurrence & Distribution & K value & Negative index \\
Set 2 & $238^{\circ} \angle 50^{\circ}$ & Fisher & 50 & Negative index \\
Set 3 & $64^{\circ} \angle 78^{\circ}$ & Fisher & 20 & Negative index \\
\hline
\end{tabular}

Note: $C_{p 3}$ is a constant and $S_{f}$ is the spacing between structural planes.

representative elementary volume $(\mathrm{REV})$. The authors in [44] introduced the index of the blockiness degree of fractured rock masses. The result indicated that the REV is 4-8 times of the spacing between discontinuities and no more than 8 times of the spacing. According to the selected maximum spacing of $6 \mathrm{~m}$, the scale of the study area is set $50 \mathrm{~m} \times 50 \mathrm{~m} \times 50 \mathrm{~m}$.

A total of 35 rock mass models with different structural plane development levels are generated using 3-Dimensional Distinct Element Codes (3DEC, Itasca Consulting [45]), as illustrated in Figure 2. To fully reflect the development of the discontinuities inside the rock mass, 5 cut planes are selected in each model. 18 scanlines are arranged on each cut plane at intervals of $10^{\circ}$. The directions of scanlines cover $0^{\circ}-360^{\circ}$. Due to the symmetry of polar coordinates, we only calculate the scanlines in the range of $0^{\circ}-180^{\circ}$. The selection of rock mass section and the layout of scanlines are displayed in Figure 3. According to the intersection of the internal structural plane of the model and the survey line, the program can automatically calculate the line density in the survey line direction. Combining the Priest-Hudson formula (equation (2), we can obtain the RQD value of the model in a certain direction.

\section{Anisotropy of the Generalized RQD}

Because of the discontinuous nature of rock masses, they are anisotropic, inhomogeneous $[46,47]$. The values of RQD in different locations of the same rock mass should be inconsistent. That is, the distribution of RQD also has anisotropy. This section introduces the concept of the generalized RQD to analyze the anisotropy of RQD based on the degree of joints development.

3.1. Generalized RQD. Selecting a reasonable threshold can expand the variation range of RQD, which plays an important role in studying the heterogeneity and anisotropy of rock mass. Therefore, the concept of generalized RQD was 


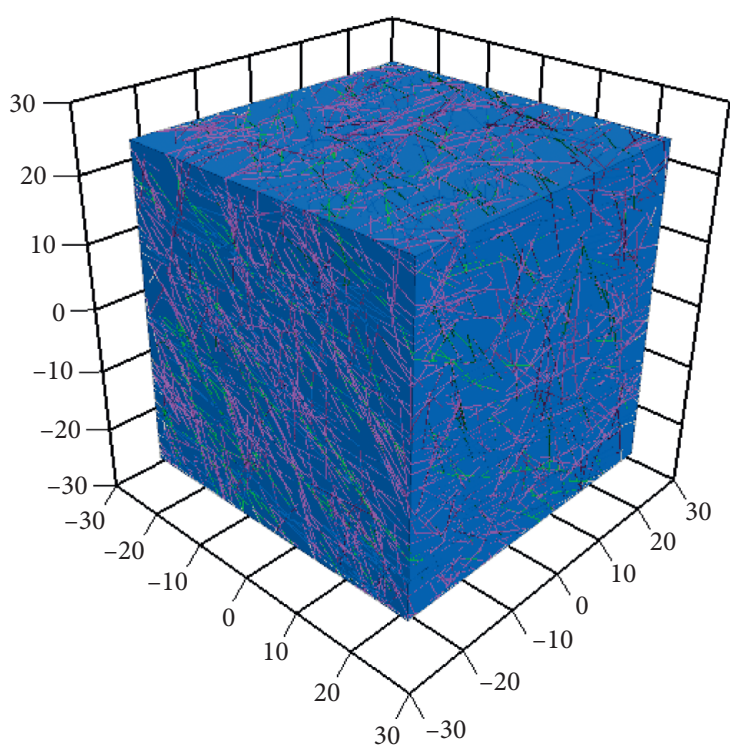

(a)

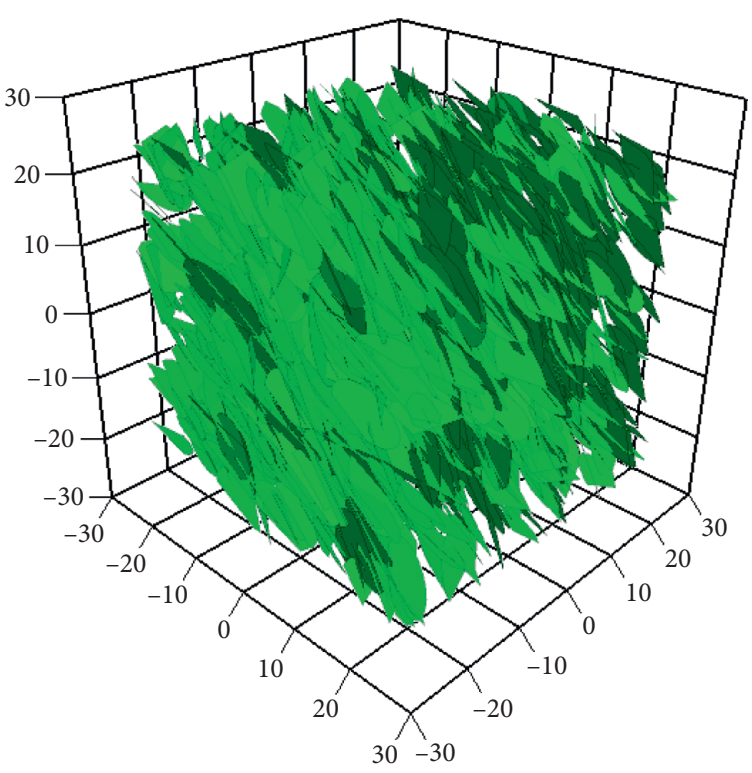

(c)

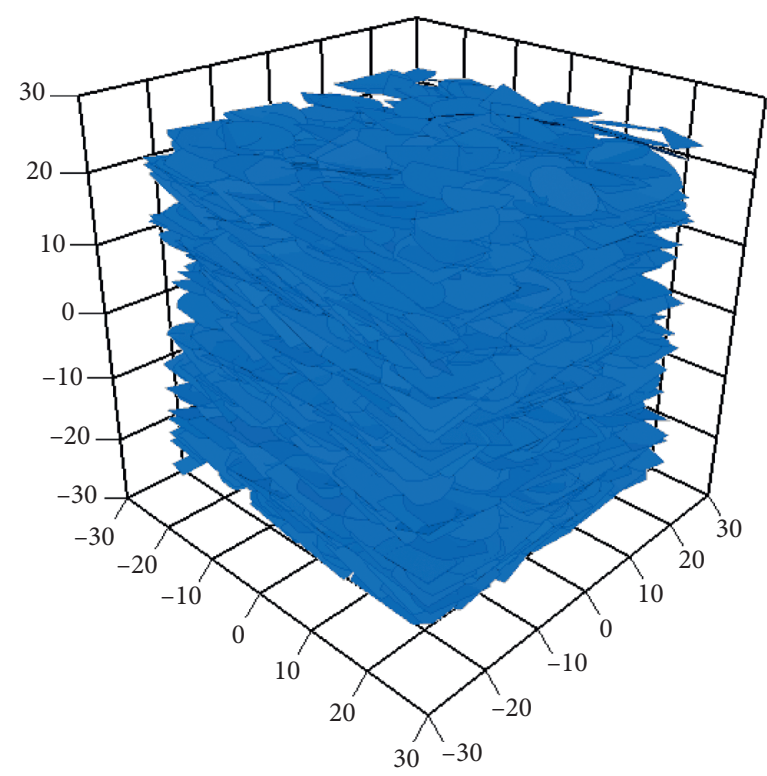

(b)

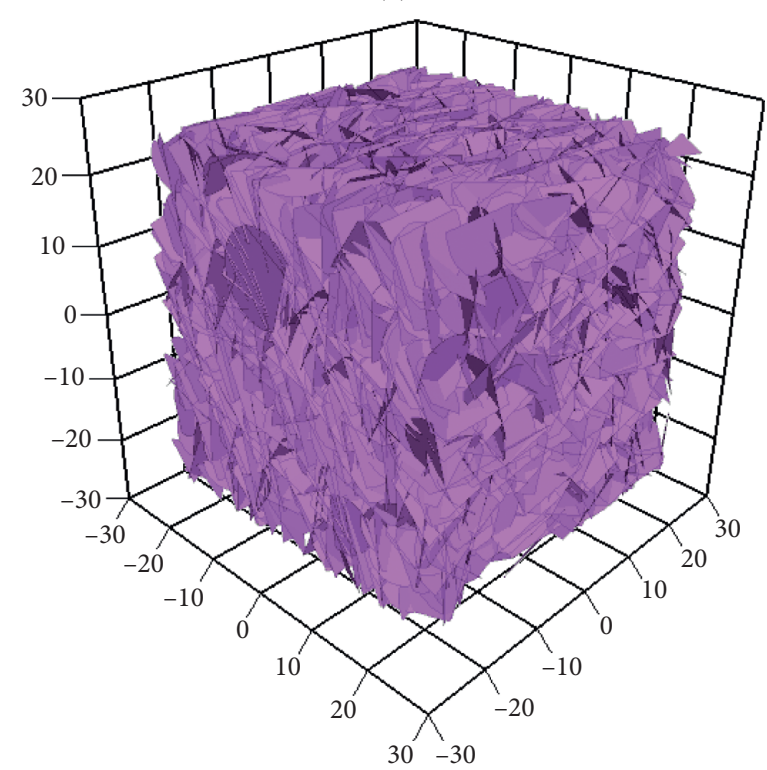

(d)

FIgURE 2: Illustration of 3D fractures (spacing: $1 \mathrm{~m}$; trace length: 8-14 m): (a) 3D plane network model; (b) dominant group set 1; (c) dominant group set 2; (d) dominant group set 3 .

introduced to maximize the variation range of the conventional RQD. For any threshold $t$, the percentage of the ratio of the sum of the length of the core length not less than $t$ along a certain scanline (borehole) to the length of the scanline (borehole) is defined as the generalized RQD [48].

According to the arrangement in Figure 3, the scanlines of the selected section are set in each model with different thresholds. The average value can be calculated based on the measurement results from those scanlines with the same direction of each section. It is regarded as the RQD inside the rock mass in a certain direction. All calculation results are drawn into a $2 \mathrm{D}$ polar diagram. As shown in Figure 4, the angle in the figure indicates the direction of the scanline and the polar radius indicates the magnitude of RQD. Due to constraints on length, Figure 4 only lists the RQD polar coordinate plane diagrams of nine models composed of extremely dense, medium dense, and extremely wide spacing with extremely low, medium, and high ductility, respectively.

3.2. Determination of Optimal Threshold. Figure 4 describes the RQD anisotropy under different structural plane development levels and different threshold conditions. As the distance remains the same, the polar coordinate plot changes within a narrow range with the ductility changes from 1-3 m 


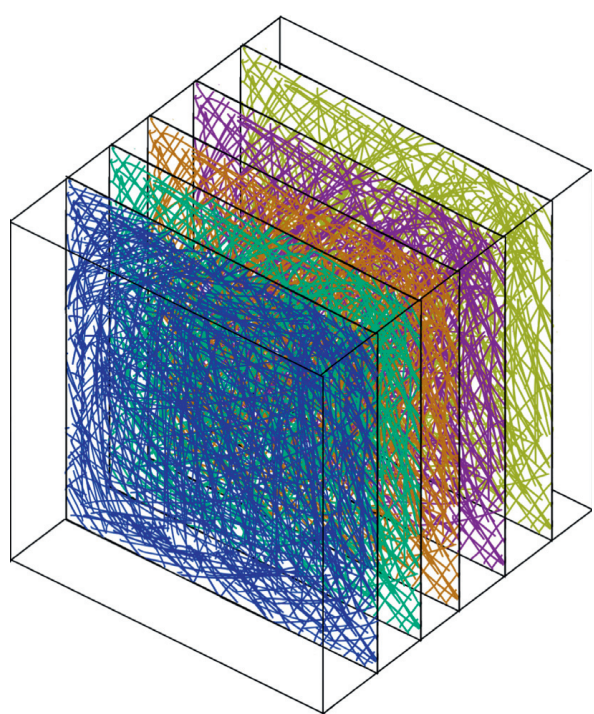

(a)

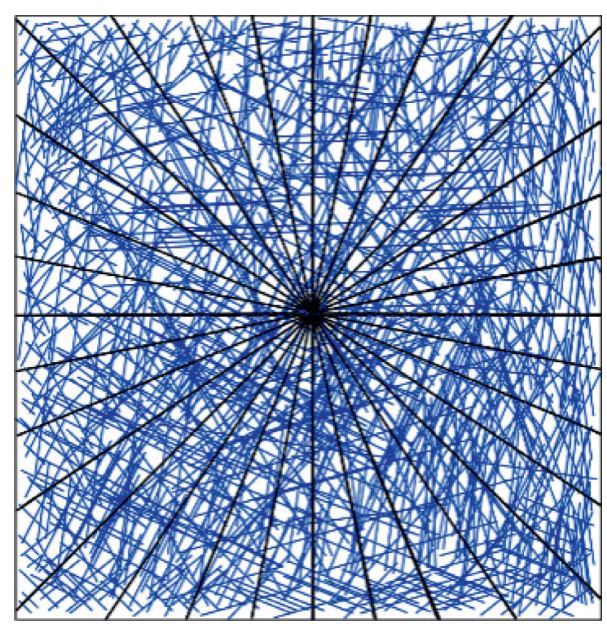

(b)

FIGURE 3: Schematic of scanline and section arrangement: (a) section's layout and (b) scanline's layout.

to $20-30 \mathrm{~m}$. As the internal ductility remains the same, however, the polar coordinate plot changes significantly with the spacing changes from $0.2 \mathrm{~m}$ to $6 \mathrm{~m}$. Hence, it is indicated that the RQD anisotropy is more sensitive to the spacing of the discontinuities. In addition, the polar coordinate plot in different models changes significantly with different thresholds, which shows that the threshold also has a greater impact on the RQD anisotropy. Therefore, the distance between the discontinuities within the model and the selected threshold are the main factors that affect the calculation results of RQD.

Since the anisotropy of the rock mass leads to the anisotropy of RQD, RQD anisotropy can reflect the anisotropy of rock mass. A reasonable threshold should be selected to maximize the variation range of RQD, which can reflect the anisotropy characteristics of rock mass. In this study, we define the optimal threshold of RQD as it can make the degree of RQD anisotropy reach the maximum in the model. Taking the model with a spacing of $1 \mathrm{~m}$ and a ductility of $8-14 \mathrm{~m}$ as an example, we set the thresholds from $0.1 \mathrm{~m}$ to $1.0 \mathrm{~m}$. The degree of the RQD anisotropy can be characterized as the range of $R Q D\left(R Q D_{\max }-R Q D_{\min }\right)$. If the range of RQD reaches the maximum, the corresponding threshold can be considered as the optimal threshold.

It is illustrated in Figure 5 that the RQD range increases first with the increase in the threshold and then decreases when reaches a certain critical value. In this model, the RQD range reaches the maximum with the threshold of $0.7 \mathrm{~m}$, which means the optimal threshold is $0.7 \mathrm{~m}$. Therefore, the conventional RQD owes obvious limitation because the calculation with the only threshold of $0.1 \mathrm{~m}$ cannot adequately characterize the anisotropy of rock mass.

Furthermore, the optimal thresholds are not the same in different models. The degree of the discontinuity's development will affect the selection of the optimal threshold. Figure 6 presents the relationship between the optimal threshold and the development of the discontinuities (including spacing and ductility) among 35 different models. It shows that the optimal threshold of RQD increases with the increase in the spacing between the discontinuities, but the ductility has no significant effect on it. With the distance of $0.2-0.5 \mathrm{~m}$, the threshold does not change with the ductility of the discontinuity. As the distance increases to $0.8-1.5 \mathrm{~m}$, the optimal threshold changes slightly with the ductility. When the distance reaches 3-6m, the optimal threshold starts to fluctuate with the change of the ductility.

Therefore, the influence of the ductility of the discontinuities on the optimal threshold of RQD is mainly related to the spacing of the discontinuities, which becomes remarkable with the increase in the spacing. Consequently, the distance between the discontinuities is the major factor for both the selection of the optimal threshold and the anisotropy of RQD.

\section{Scale Effect of the Generalized RQD}

Engineers usually calculate the RQD according to the length of the core in the borehole, ignoring the impact of the drilling depth on the RQD value. In fact, the true RQD of rock mass at any drilling depth (the length of the scanline) cannot be obtained $[49,50]$. Thus, the issue is discussed using 3D fracture network simulation. The scale effect of the generalized RQD can be analyzed by changing the length of the scanlines.

Generally, engineering geological drillings are vertical because of the geological environment and drilling technology. Therefore, the scanlines are arranged as boreholes in the generated rock mass model, which are perpendicular to the $x$ o $y$ plane. A total of 121 scanlines are shown in Figure 7. The scanline is arranged every $5 \mathrm{~m}$ to get enough data.

Priest and Hudson [27] suggested a theoretical equation related to the calculation of the generalized RQD (equation 


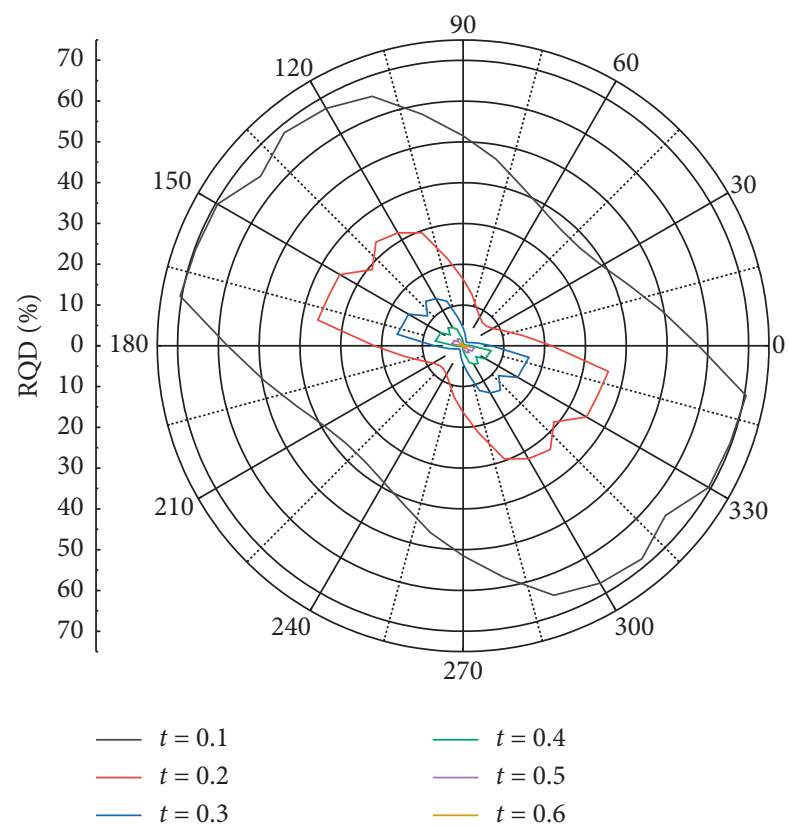

(a)

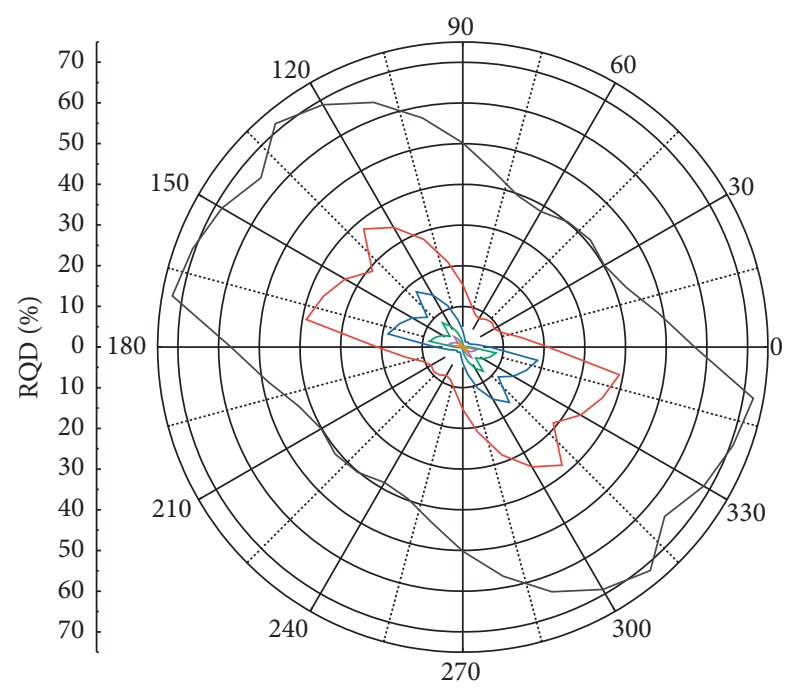

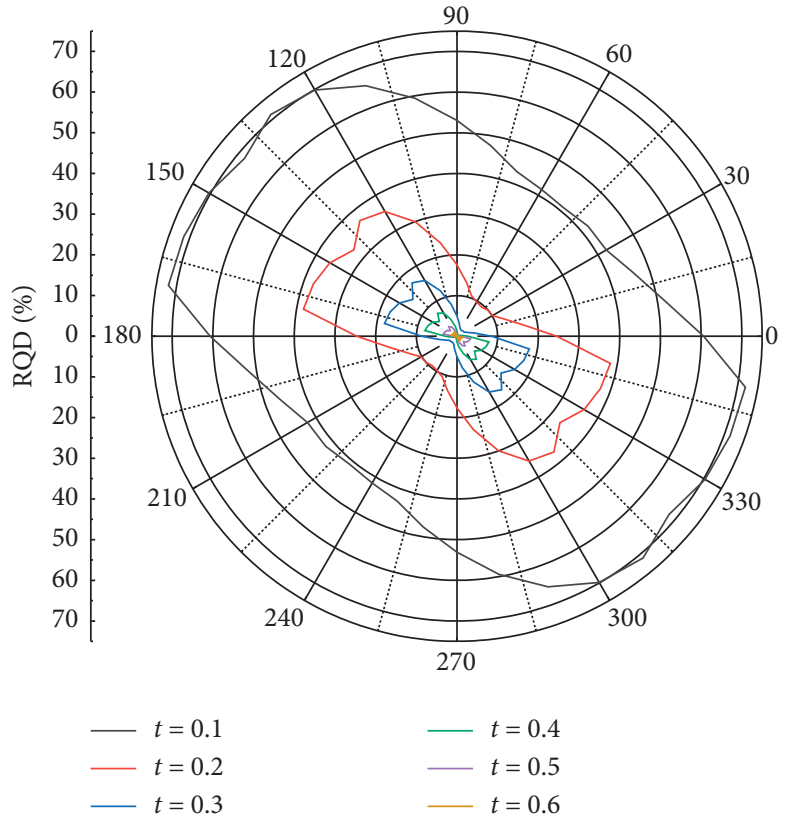

(b)
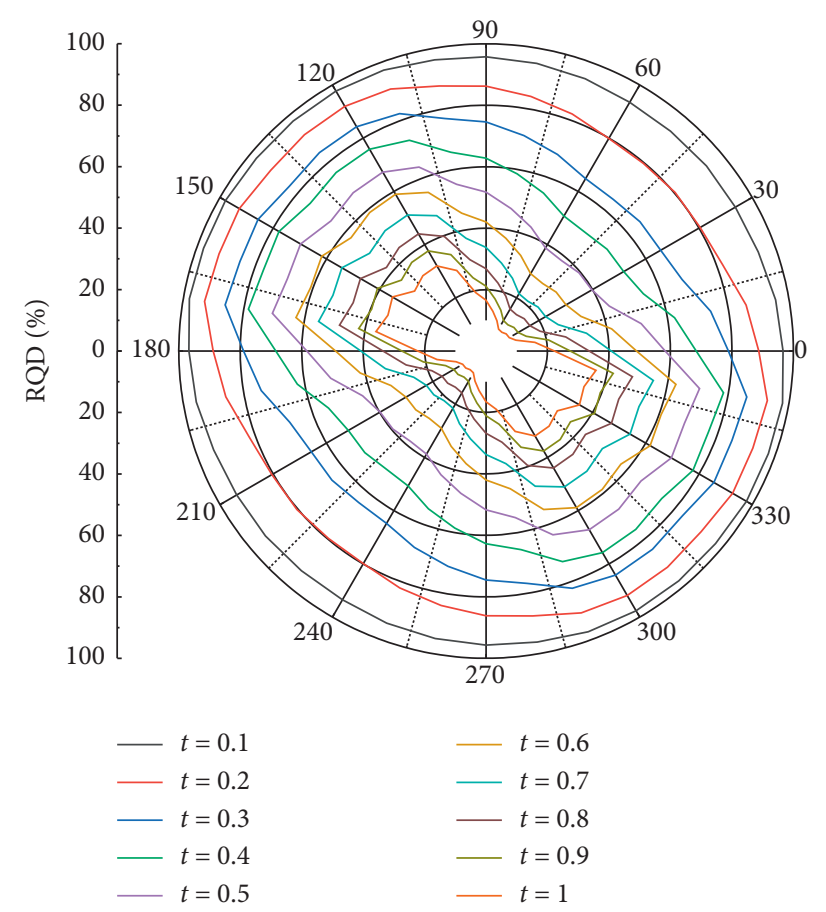

(d)

FIgURE 4: Continued. 

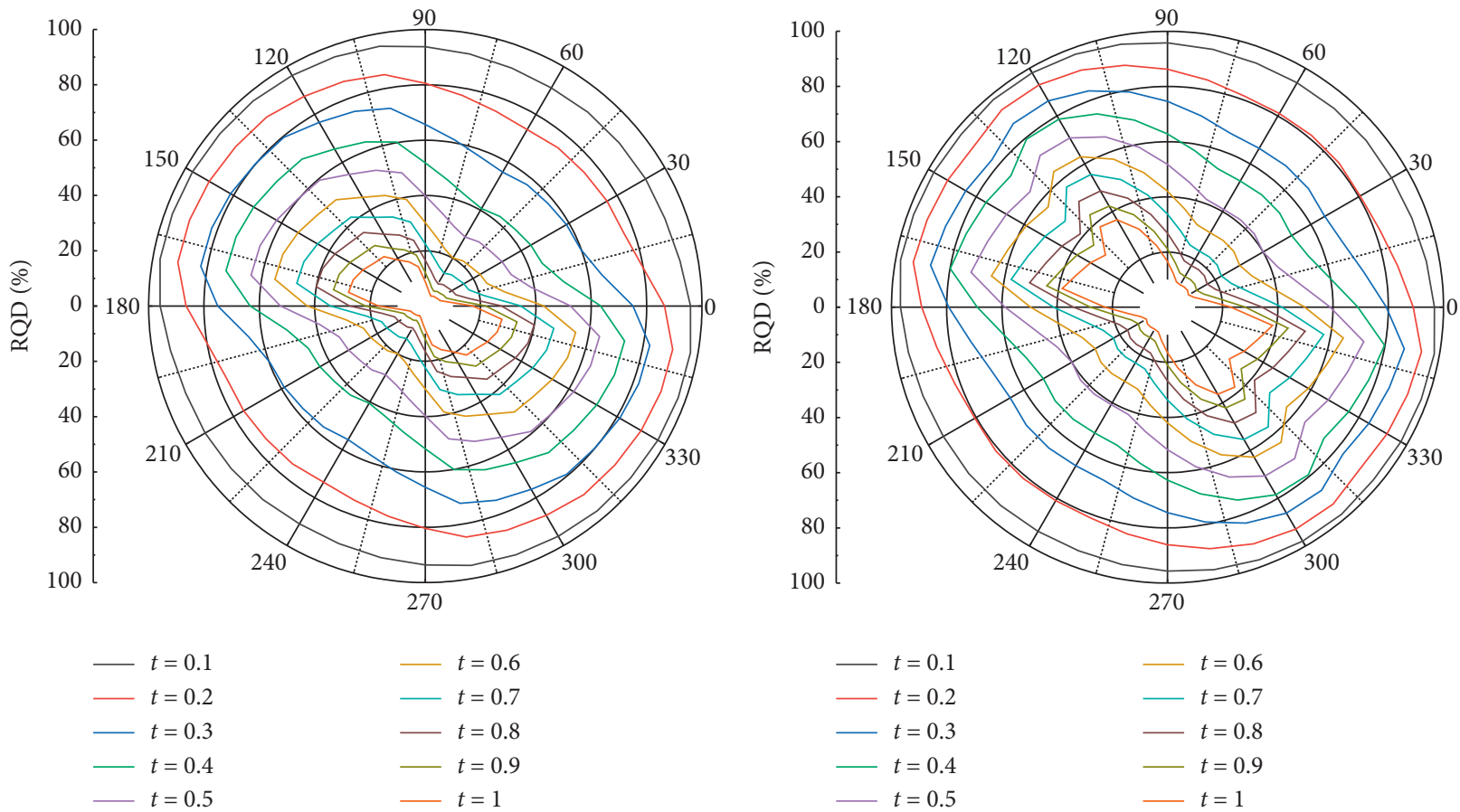

(e)
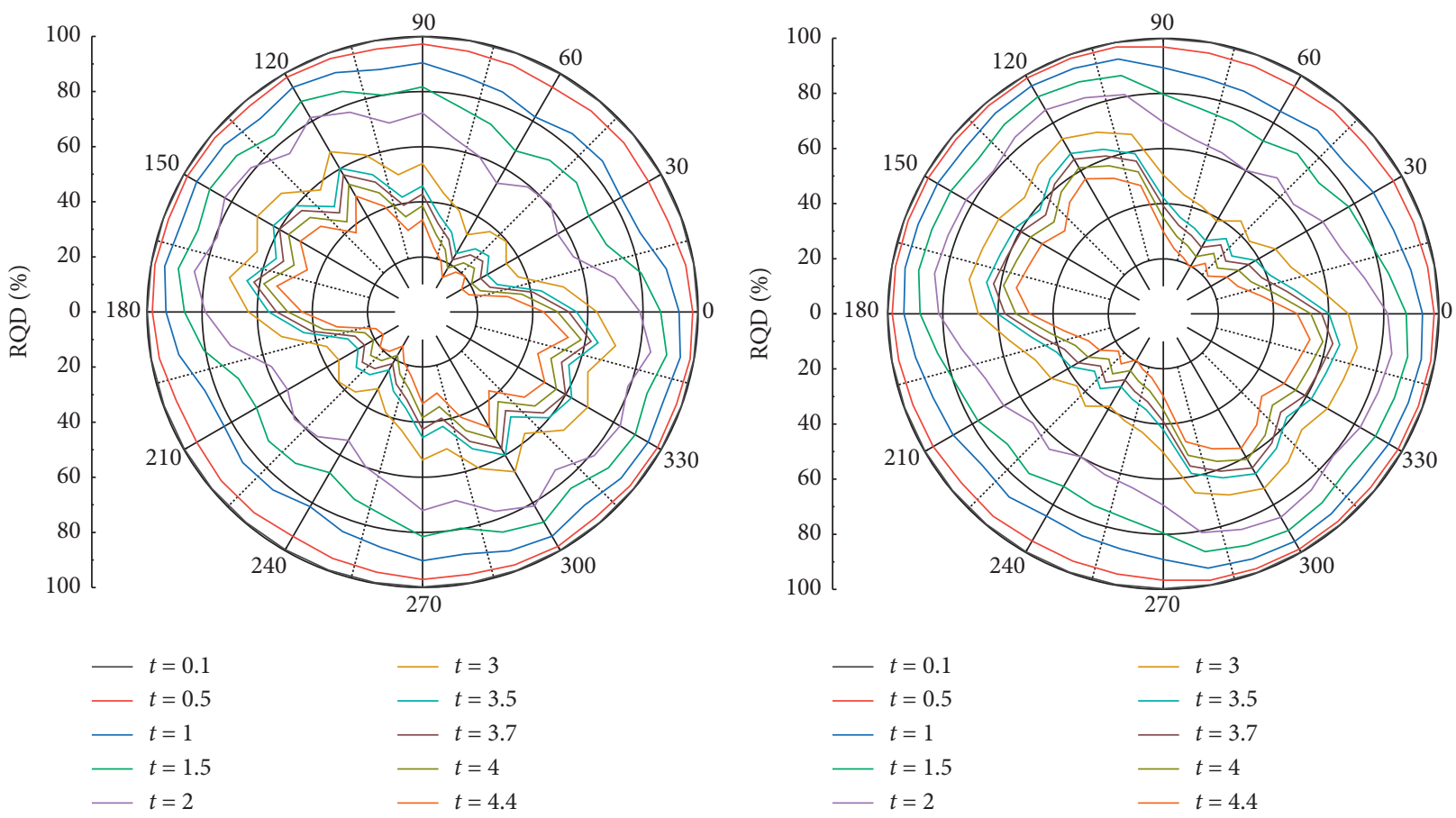

(g)

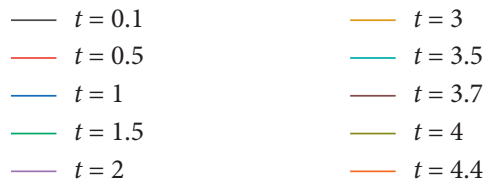

(h)

FIgure 4: Continued. 


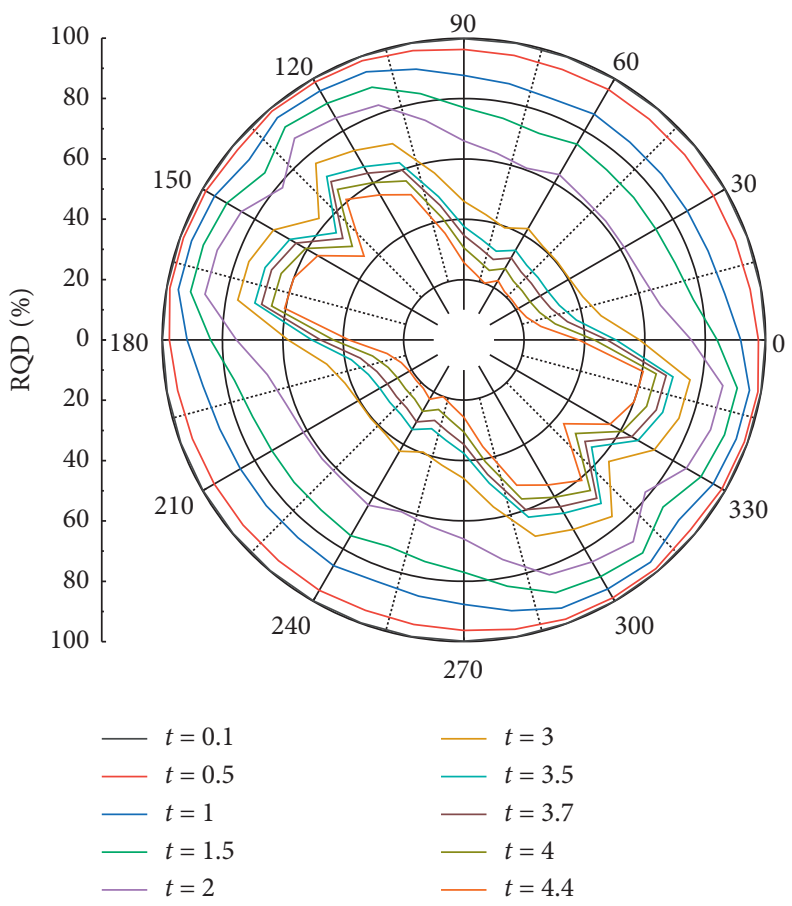

(i)

FIGURE 4: Generalized RQD with various thresholds in different directions of diverse models: (a) spacing 0.2 , scalability: 1-3; (b) spacing 0.2, scalability: $8-14$; (c) spacing 0.2 , scalability: 20-30; (d) spacing 1, scalability: $1-3$; (e) spacing 1, scalability: 8-14; (f) spacing 1, scalability: 20-30; (g) spacing 6, scalability: 1-3; (h) spacing 6, scalability: 8-14; (i) spacing 6, scalability: 20-30.

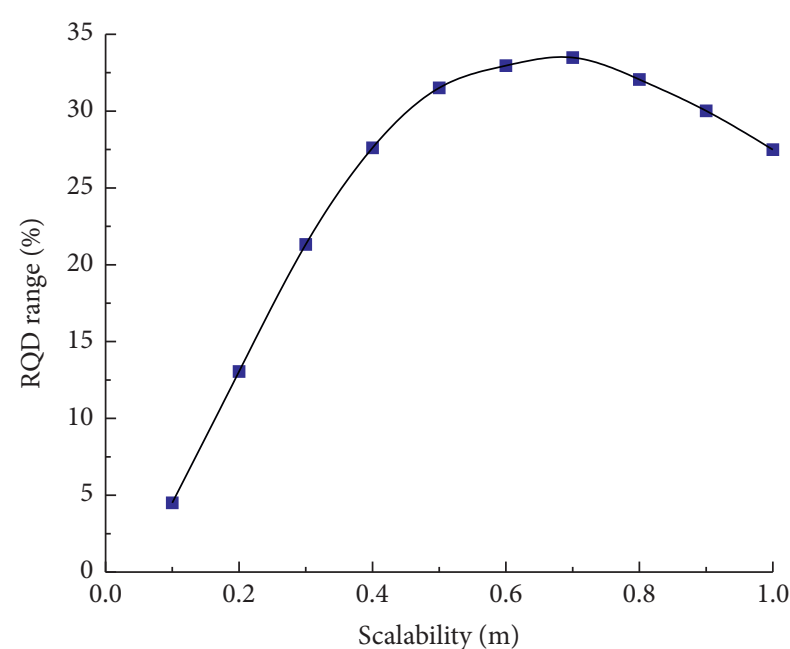

FIGURE 5: RQD range for different thresholds (spacing: $1 \mathrm{~m}$; trance length: $8-14 \mathrm{~m}$ ).

(3)), with respect to the hypothesis of negative exponential distribution of joint spacing. Equation (2) was proposed with regard to the effect of the scanline length. Another possible more realistic expression for the generalized RQD was also suggested by [51]. Compared with equation (3), the calculation of equation (4) is concerned more about the influence of short scanline on the RQD result. In these equations, $\lambda$ is the density of the discontinuities, $t$ is the calculation threshold, and $L$ is the length of the scanline.

$$
\begin{aligned}
& \mathrm{RQD}=100\left[(1+\lambda t) e^{-\lambda t}-(1+\lambda L) e^{-\lambda L}\right], \\
& \mathrm{RQD}=\frac{100\left[(1+\lambda t) e^{-\lambda t}-(1+\lambda L) e^{-\lambda L}\right]}{1-e^{-\lambda L}-\lambda L e^{-\lambda L}} .
\end{aligned}
$$

Five typical models with intervals of $0.2,0.5,0.8$, and $1 \mathrm{~m}$ were selected as examples. The values of the generalized RQD with different lengths of the scanline are calculated according to equation (4) and the optimal thresholds of each model. The variation curves of the generalized RQD are plotted in Figure 8. It is assumed that the true RQD can be obtained under the full length of scanline, i.e., $50 \mathrm{~m}$ in this study. Then, the rate of RQD change is brought forward to compare the generalized RQD under each scanline length in different models with that corresponding to the scanline length of $50 \mathrm{~m}$. The relationship between the rate of RQD change and the length of the scanline is shown in Figure 9.

The various models indicate that the generalized RQD converges with the increase in the scanline length, which has an obvious scale effect. The RQD result can represent the rock mass quality more accurately under the longer scanline. However, the convergence of the generalized RQD in different models is not consistent. When the spacing is $0.2 \mathrm{~m}$, the RQD changes from large to small and then to converge as the length of the scanline increases. As the distance between the discontinuities increases, the change of RQD increases first and then decreases to convergence with the length of the scanline. The ductility of the discontinuities also affects the convergence of the RQD. The rate of the RQD change in 


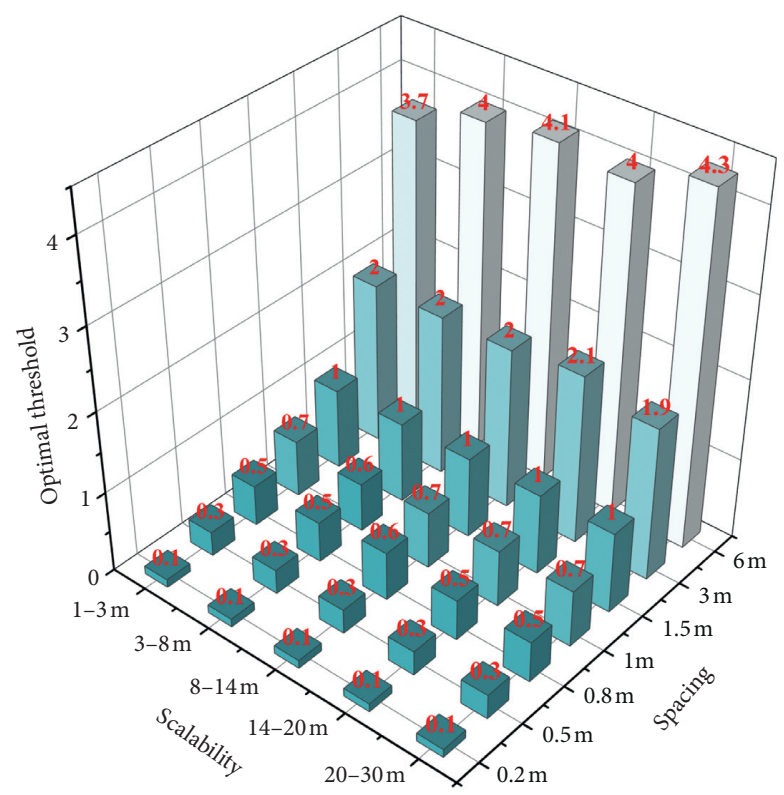

Figure 6: Optimal threshold in different models.

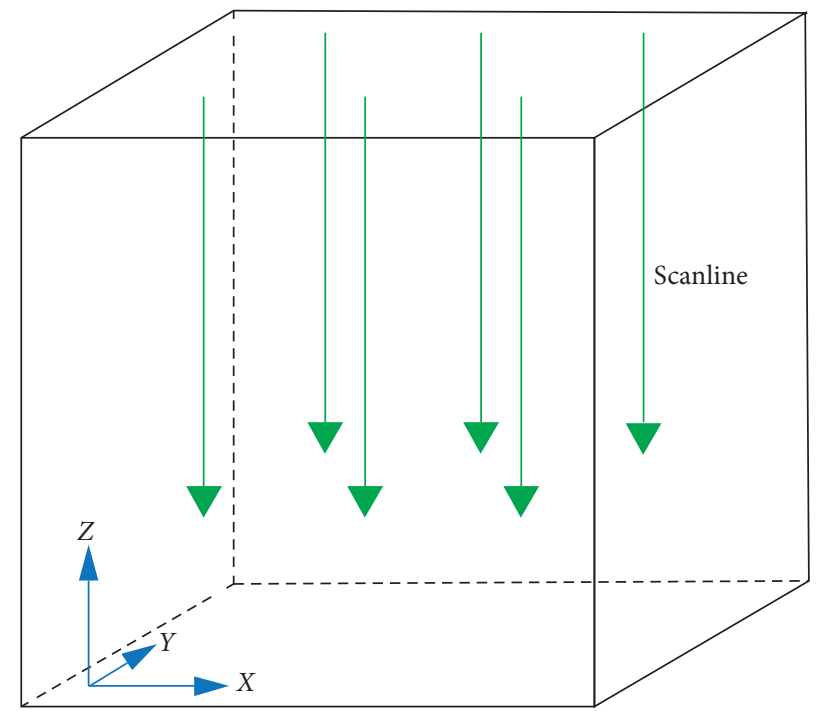

FIgURE 7: Schematic of vertical scanline setting.

each model increases as the ductility increases. It is indicated that the convergence length of scanline is positively associated with the ductility of the discontinuities.

For those rock masses without a recognizable structural pattern, it is difficult to determine the real RQD. When the number of scanlines is large enough, the average value of RQD can be close to the real RQD of rock mass infinitely. Therefore, the average RQD obtained from 121 scanlines with full length ( $50 \mathrm{~m}$ in this study) is assumed to be the real RQD. Subsequently, the average value obtained from different lengths of scanlines, which is recorded as RQD ${ }^{\#}$, is regarded as the undetermined RQD of the rock mass. This study defines the variation rate of RQD as the difference between $\mathrm{RQD}^{\#}$ and RQD. With respect to the engineering requirement, it is considered to meet the research requirements when the variation rate of RQD is not greater than $2 \%$, which is described in equation (5). We defined the $\mathrm{RQD}^{\#}$ in a certain scanline satisfied the inequality as the convergence RQD value, and the corresponding scanline length is the convergence length of the scanline.

$$
\left|\frac{\mathrm{RQD}^{\#}-\mathrm{RQD}}{\mathrm{RQD}}\right| \leq 2 \% \text {. }
$$

Table 3 tabulates the shortest convergence length of the scanline and the convergence RQD value that meet equation (5) requirements of each model. The degree of joint development distinctly affects the convergence length of the 
Spacing: $0.2 \mathrm{~m}$

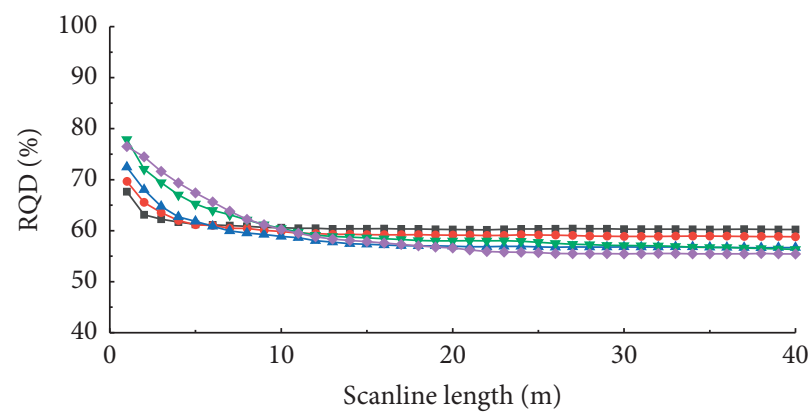

(a)

Spacing: $0.8 \mathrm{~m}$

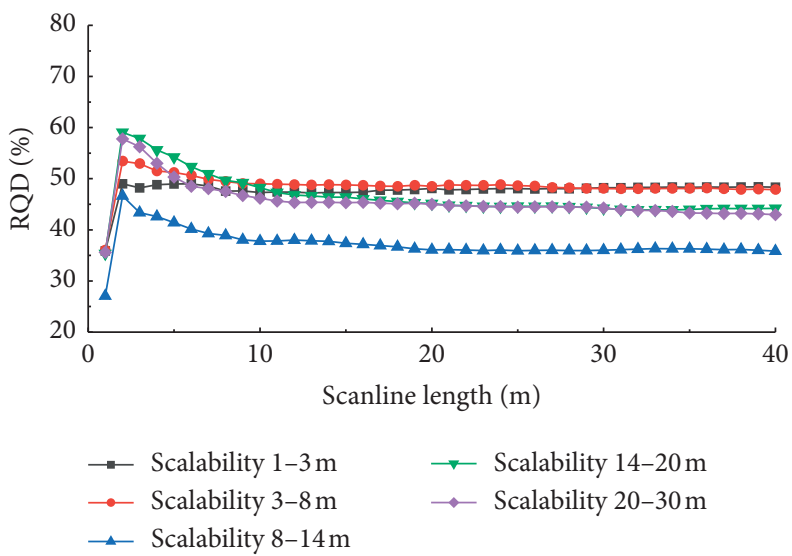

(c)

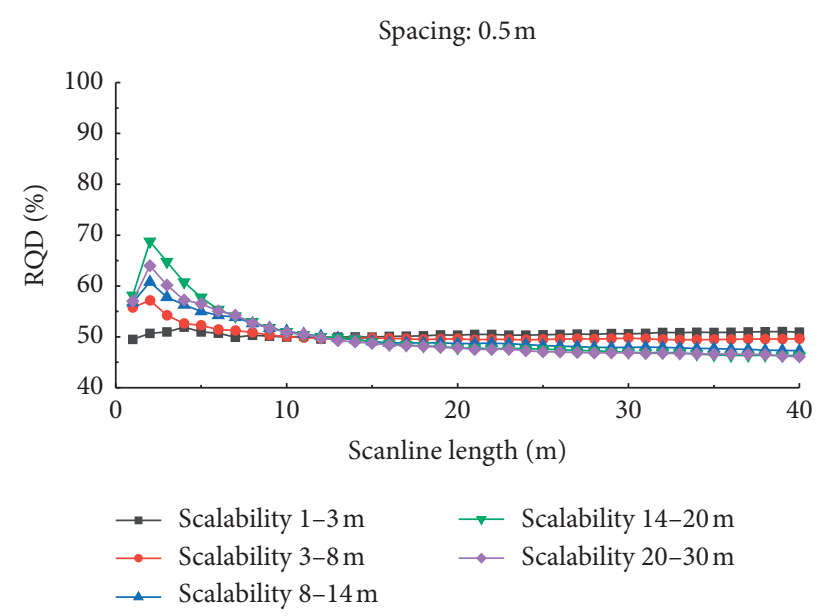

(b)

Spacing: $1 \mathrm{~m}$

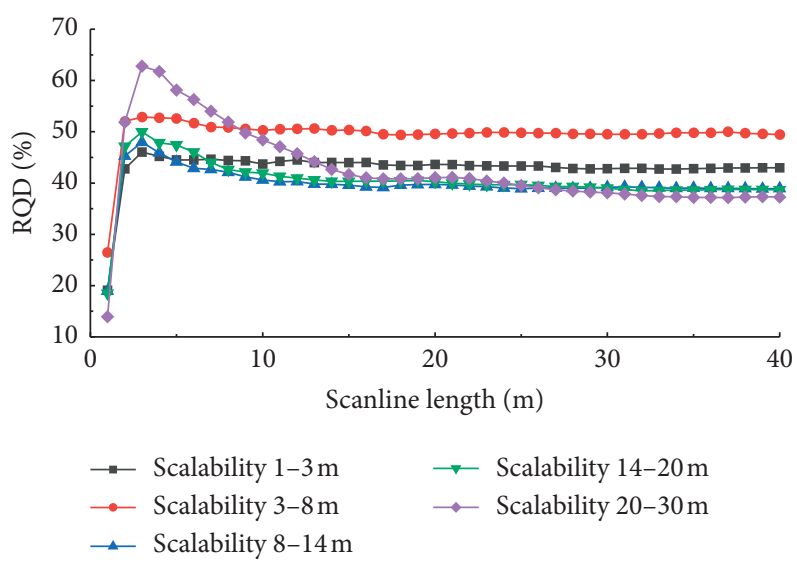

(d)

FIgURE 8: Variation of RQD values with scanline length.

Spacing: $0.2 \mathrm{~m}$

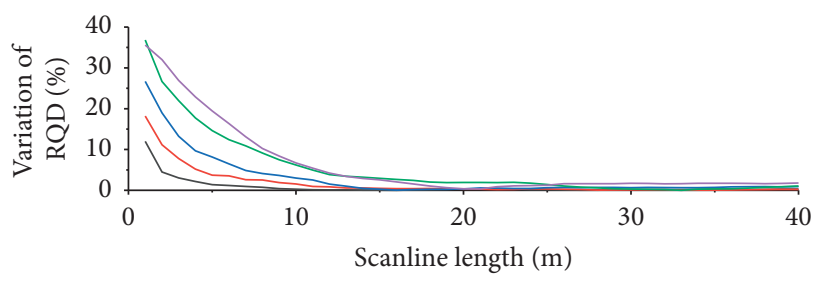

$\begin{array}{ll}\text { Scalability } 1-3 \mathrm{~m} & - \text { Scalability } 14-20 \mathrm{~m} \\ \text { Scalability } 3-8 \mathrm{~m} & - \text { Scalability } 20-30 \mathrm{~m}\end{array}$

(a)

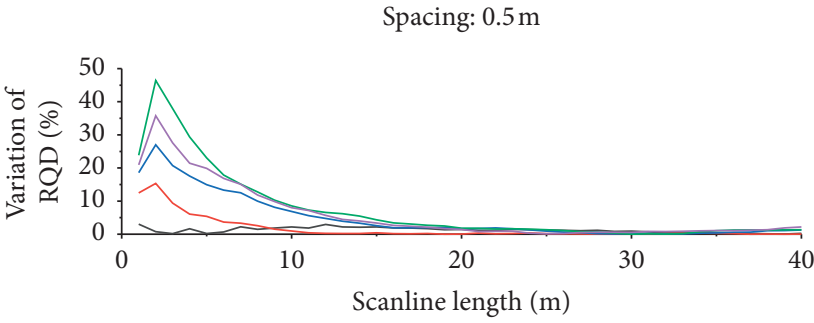

$\begin{array}{ll}\text { Scalability } 1-3 \mathrm{~m} & - \text { Scalability } 14-20 \mathrm{~m} \\ \text { Scalability } 3-8 \mathrm{~m} & - \text { Scalability } 20-30 \mathrm{~m}\end{array}$

(b)

Figure 9: Continued. 


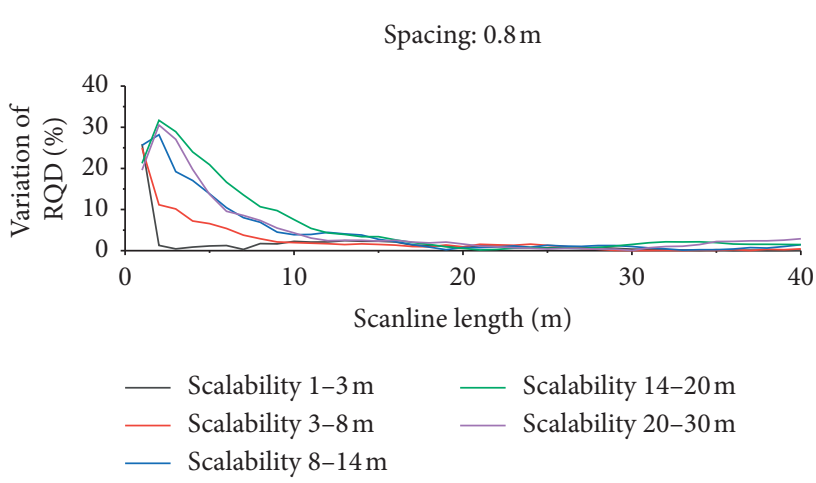

(c)

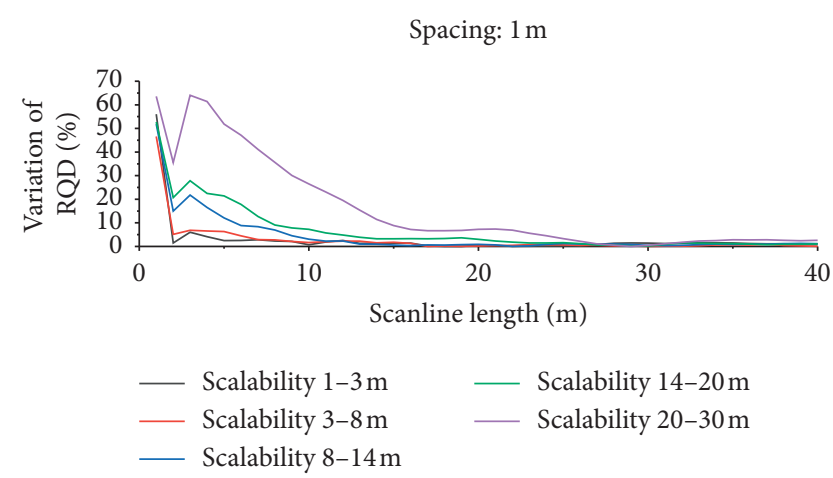

(d)

FIGURE 9: Variation rate of RQD values with scanline length.

TABLE 3: Convergence scanline lengths and convergence RQD values of different models.

\begin{tabular}{|c|c|c|c|c|c|c|c|c|}
\hline \multirow[b]{2}{*}{$\begin{array}{l}\text { Joint } \\
\text { trace } \\
\text { length }\end{array}$} & \multicolumn{2}{|c|}{$0.2 \mathrm{~m}$} & \multicolumn{2}{|c|}{$0.5 \mathrm{~m}$} & \multicolumn{2}{|c|}{$0.8 \mathrm{~m}$} & \multicolumn{2}{|c|}{$1 \mathrm{~m}$} \\
\hline & $\begin{array}{c}\text { Convergence } \\
\text { line length } \\
(\mathrm{L} / \mathrm{m})\end{array}$ & $\begin{array}{c}\text { Convergence } \\
\text { RQD (\%) }\end{array}$ & $\begin{array}{c}\text { Convergence } \\
\text { line length } \\
(\mathrm{L} / \mathrm{m})\end{array}$ & $\begin{array}{l}\text { Convergence } \\
\text { RQD (\%) }\end{array}$ & $\begin{array}{c}\text { Convergence } \\
\text { line length } \\
(\mathrm{L} / \mathrm{m})\end{array}$ & $\begin{array}{c}\text { Convergence } \\
\text { RQD (\%) }\end{array}$ & $\begin{array}{c}\text { Convergence } \\
\text { line length } \\
(\mathrm{L} / \mathrm{m})\end{array}$ & $\begin{array}{c}\text { Convergence } \\
\text { RQD (\%) }\end{array}$ \\
\hline $1-3 \mathrm{~m}$ & 5 & 61.2 & 7 & 49.9 & 10 & 47.3 & 13 & 44 \\
\hline $3-8 \mathrm{~m}$ & 9 & 60.1 & 9 & 50.3 & 10 & 49 & 14 & 50.3 \\
\hline $8-14 \mathrm{~m}$ & 12 & 58 & 16 & 48.8 & 17 & 37 & 13 & 39.8 \\
\hline $14-20 \mathrm{~m}$ & 19 & 58 & 20 & 47.8 & 18 & 45.5 & 22 & 39.8 \\
\hline 20-30 m & 17 & 57.3 & 19 & 47.9 & 20 & 45 & 27 & 38.7 \\
\hline
\end{tabular}

scanline. The convergence length generally exhibits a slight increase with the increase in the spacing. However, the increase is not uniform in each model. Compared with the spacing, the ductility greatly influences the convergence length of the scanline. With the same spacing, the convergence length in different models increases with the increase in ductility.

According to the combined results of Figures 7 and 8 and Table 3, the generalized RQD has an obvious scale effect. The RQD result is more stable with respect to the longer measuring line, and it can represent the quality of the entire rock mass more accurately. In addition, a longer scanline is needed to obtain a reliable RQD value for a heavily fractured rock mass.

\section{Conclusions}

This study used 3D structural plane network simulation technology to generate 35 rock mass models with different structural plane spacing and ductility, arranged survey lines inside the model instead of drilling holes, obtained enough values of the generalized RQD, and studied the relationship between the generalized RQD characteristics and the development of discontinuities. The specific conclusions are as follows:

(1) The traditional method using $0.1 \mathrm{~m}$ as the only threshold for RQD has significant limitations. It cannot fully reflect the anisotropy and scale effect of the rock mass. The generalized RQD concept is introduced, and the optimal threshold is obtained based on the degree of RQD anisotropy. The results show that the degree of joint development affects the selection of the optimal threshold. The optimal threshold increases with the increase in the spacing, while of the discontinuities have a minimal influence on the selection of the optimal threshold. However, the influence degree of the ductility shows an increasing trend with the increase in the spacing.

(2) The degree of joint development has a significant effect on RQD anisotropy [52]. When the spacing is larger, the rock mass has higher anisotropy, while the ductility has a minimal effect on RQD anisotropy, which can even be ignored. Therefore, if the discontinuities with a larger spacing are encountered in the practical engineering, the number of drillings should be increased so that the RQD calculation can fully reflect the anisotropy of the rock mass.

(3) The generalized RQD has an obvious scale effect. The longer the scanline, the more stable the RQD obtained, and the more accurately it can represent the quality of the rock mass. In addition, when the discontinuities are highly developed in the rock mass, the scale effect of RQD is more obvious, and a longer scanline is needed to obtain a real RQD. Therefore, the sufficient length of the scanline is necessary to obtain stable and reliable RQD. Particularly, in heavily fractured rock masses, the length 
of the scanline should be appropriately increased to ensure the accuracy of RQD [53].

\section{Data Availability}

The data used to support the findings of this study are included within the article.

\section{Conflicts of Interest}

The authors declare that there are no conflicts of interest in the publication of this article.

\section{Acknowledgments}

This study was partially funded by the Zhejiang Provincial Natural Science Foundation of China (Grant no. LQ18D020001) and National Natural Science Foundation of China (Grant no. 42007254), and this support is gratefully acknowledged.

\section{References}

[1] H. Bao, Y. Zhai, H. Lan et al., "Distribution characteristics and controlling factors of vertical joint spacing in sand-mud interbedded strata," Journal of Structural Geology, vol. 128, Article ID 103886, 2019.

[2] H. Bao, X. Xu, H. Lan et al., "A new joint morphology parameter considering the effects of micro-slope distribution of joint surface," Engineering Geology, vol. 275, Article ID 105734, 2020a.

[3] M. Huang, C. Hong, J. Chen et al., "Prediction of the peak shear strength of rock joints based on BP neural network," International Journal of Geomechanics, vol. 21, no. 6, Article ID 04021085, 2021.

[4] X. Zhang, H. Lin, Y. Wang, and Y. Zhao, "Creep damage model of rock mass under multi-level creep load based on spatio-temporal evolution of deformation modulus," Archives of Civil and Mechanical Engineering, vol. 21, 2021.

[5] D. U. Deere, "Technical description of rock cores for engineering purposes," Rock Mechanics and Engineering Geology, vol. 1, no. 1, pp. 18-22, 1963.

[6] D. U. Deere, A. J. Hendron, F. D. Patton, and E. J. Cording, "Design of surface and near surface construction in rock," in Proceedings of the 8th US Symposium Rock Mech Fail Break Rock, pp. 237-302, Minneapolis, MN, USA, September 1966.

[7] Z. T. Bieniawski, "Engineering classification of jointed rock masses," Transactions of the South African Institution of Civil Engineers, vol. 15, no. 12, pp. 335-343, 1973.

[8] Z. T. Bieniawski, "Rock mass classification in rock engineering," in Proceedings of the symposium on exploration and rock engineering, vol. 2, pp. 97-106, Johannesburg, South Africa, November 1976.

[9] Z. T. Bieniawski, "The geomechanics classification in rock engineering applications," in Proceedings of the 4th int congress on rock mechanics, vol. 3, pp. 41-48, Montreux, Switzerland, September 1979.

[10] Z. T. Bieniawski, The Rock Mass Rating (Rmr) System In Engineering Practice Rock Classification Systems for Engineering Purposes, L. Kirkaldie, Ed., pp. 17-34, American Society for Testing Materials, Philadelphia, PA, 1988.
[11] N. Barton, R. Lien, and J. Lunde, "Engineering classification of rock masses for the design of tunnel support," Rock Mechanics, vol. 6, no. 4, pp. 189-236, 1974.

[12] E. Hoek and E. T. Brown, Underground Excavations in Rock, Institution of Mining and Metallurgy, London, England, 1980.

[13] E. Hoek and J. W. Bray, Rock Slope Engineering, The Institution of Mining and Metallurgy, London, England, 3rd edition, 1981.

[14] D. U. Deere, Geological Considerations, Chapter 1, Rock Mechanics in Engineering Practice, K. G. Stagg and O. C. Zienkiewicz, Eds., pp. 1-20, Wiley, London, England, 1968.

[15] J. A. Hudson and S. D. Priest, "Discontinuity frequency in rock masses," International Journal of Rock Mechanics and Mining Science \& Geomechanics Abstracts, vol. 20, no. 2, pp. 73-89, 1983.

[16] J. P. Harrison, "Selection of the threshold value in RQD assessments," International Journal of Rock Mechanics and Mining Sciences, vol. 36, no. 5, pp. 673-685, 1999.

[17] J. P. Chen, J. H. Fan, and D. Liu, "Review and prospect on the application and research of RQD," Rock and Soil Mechanics, vol. 26, no. S2, pp. 249-252, 2005, in Chinese.

[18] P. Pells, Z. Bieniawski, S. Hencher et al., "RQD: time to rest in peace," Canadian Geotechnical Journal, vol. 54, pp. 825-834, 2017.

[19] Q. Chen, T. Yin, and H. Jia, "Selection of optimal threshold of generalized rock quality designation based on modified blockiness index," Advances in Civil Engineering, no. 1, pp. 1-11, 2019.

[20] S. Y. Choi and H. D. Park, "Variation of rock quality designation (RQD) with scanline orientation and length: a case study in Korea," International Journal of Rock Mechanics and Mining Sciences, vol. 41, no. 2, pp. 207-221, 2004.

[21] L. Li, S. Ouellet, and M. Aubertin, "An improved definition of rock quality designation, RQDc," in Proceedings of the 3 rd CANUS Rock Mechanics Symposium ROCKENG09, M. Diederichs and G. Grasselli, Eds., pp. 1-10, Toronto, Canada, May 2009.

[22] B. Wang, H. M. Tang, W. X. Jian et al., "Application of 3d network modeling rock mass discontinuities to evaluating rock mass quality for dam foundation," Rock and Soil Mechanics, vol. 27, no. 4, pp. 594-596, 2006, in Chinese.

[23] J. Zheng, X. Yang, Q. Lü et al., "A new perspective for the directivity of Rock Quality Designation (RQD) and an anisotropy index of jointing degree for rock masses," Engineering Geology, vol. 240, pp. 81-94, 2018.

[24] Z. Sen and E. A. Eissa, "Rock quality charts for long-normally distributed block size," Int J Rock Mech Min Sci Geomech Abstr Pergamon, vol. 29, no. 1, pp. 1-12, 1992.

[25] A. Palmström, "Measurements of and correlations between block size and rock quality designation (RQD)," Tunnelling and Underground Space Technology, vol. 20, no. 4, pp. 362377, 2005.

[26] Y. Chen, H. Lin, X. Ding, and S. Xie, "Scale effect of shear mechanical properties of non-penetrating horizontal rocklike joints," Environmental Earth Sciences, vol. 80, 2021.

[27] S. D. Priest and J. A. Hudson, "Discontinuity spacings in rock," International Journal of Rock Mechanics and Mining Science \& Geomechanics Abstracts, vol. 13, no. 5, pp. 135-148, 1976.

[28] S. G. Du, S. F. Xu, S. F. Yang et al., "Application of rock quality designation (RQD) to engineering classification of rocks," Journal of Engineering Geology, vol. 8, no. 3, pp. 351-356, 2000, in Chinese. 
[29] G. X. Wang, X. D. Wang, and S. F. Xiao, "Study on applying broad sense RQD to anisotropy of rock mass," Journal of Jilin University (Earth Science Edition), vol. 32, no. 3, pp. 258-260, 2002a, in Chinese.

[30] W. Zhang, J. P. Chen, X. Q. Yuan et al., "Study of size effect and spatial effect of RQD for rock masses based on threedimensional fracture network," Chinese Journal of Rock Mechanics and Engineering, vol. 31, no. 7, pp. 1437-1445, 2012a, in Chinese.

[31] A. Azimian, "A new method for improving the RQD determination of rock core in borehole," Rock Mechanics and Rock Engineering, vol. 49, no. 4, pp. 1559-1566, 2016.

[32] H. S. Lee and T. F. Cho, "Hydraulic characteristics of rough fractures in linear flow under normal and shear load," Rock Mechanics and Rock Engineering, vol. 35, no. 4, pp. 299-318, 2002.

[33] B. Noetinger and N. Jarrige, "A quasi-steady state method for solving transient Darcy flow in complex $3 \mathrm{~d}$ fractured networks accounting for matrix to fracture flow," Journal of Computational Physics, vol. 231, no. 1, pp. 23-38, 2015.

[34] Austria Startup Company, ShapeMetriX3D System Manuals. Shengyang, Europe and China Co. LTD., Shengyang, China, 2008.

[35] D. Z. Gu, Foundation of Engineering Geomechanics of Rock Mass, Science Press, Beijing, China, 2008.

[36] B. H. Kim, M. Cai, P. K. Kaiser et al., "Estimation of block sizes for rock masses with non-persistent joints," Rock Mechanics and Rock Engineering, vol. 40, no. 2, pp. 169-192, 2006.

[37] ISRM, "Suggested methods for the quantitative description of discontinuities in rock masses," International Journal of Rock Mechanics and Mining Science \& Geomechanics Abstracts, vol. 15, no. 6, pp. 319-368, 1978.

[38] W. Zhang, J. P. Chen, C. C. Niu et al., "Determination of RQD and number of optimum scanlines based on three-dimensional fracture network," Chinese Journal of Geotechnical Engineering, vol. 35, no. 2, pp. 321-327, 2013, in Chinese.

[39] J. Zheng, J. Deng, X. Yang et al., “An improved Monte Carlo simulation method for discontinuity orientations based on Fisher distribution and its program implementation," Computers and Geotechnics, vol. 61, pp. 266-276, 2014.

[40] F. Wu, J. Wu, H. Bao, B. Li, Z. Shan, and D. Kong, "Advances in statistical mechanics of rock masses and its engineering applications," Journal of Rock Mechanics and Geotechnical Engineering, vol. 13, no. 1, pp. 22-45, 2021.

[41] W. S. Dershowitz, Rock Joint Systems, Ph.D. Dissertation, Ph.D. Dissertation Submitted to Massachusetts Institute of Technology, Cambridge, MA, USA, 1984.

[42] W. S. Dershowitz and H. Herda, "Interpretation of fracture spacing and intensity," in Proceedings of the 33" US Rock Mechanics Symposium, vol. 33, pp. 757-766, NM, USA, June 1992.

[43] Y. H. An and Q. Wang, "Analysis of representative element volume size based on 3d fracture network," Rock and Soil Mechanics, vol. 33, no. 12, pp. 3775-3780, 2013, in Chinese.

[44] L. Xia, X. F. Liu, and Q. C. Yu, "Determining representative elementary volume of fractured rock mass based on blockiness analysis," Rock and Soil Mechanics, vol. 31, no. 12, pp. 3991-3996, 2010, in Chinese.

[45] Itasca Consulting Group, Inc, Distinct Element Method, 2017, http://www.itascacg.com/software/products/3dec/distinct-elementmethod $>$.

[46] H. Bao, K. Zhang, C. Yan et al., "Excavation damaged zone division and time-dependency deformation prediction: a case study of excavated rock mass at Xiaowan hydropower station,” Engineering Geology, vol. 272, Article ID 105668, 2020 b.

[47] F. Wu, Y. Deng, J. Wu et al., "Stress-strain relationship in elastic stage of fractured rock mass," Engineering Geology, vol. 268, Article ID 105498, 2020.

[48] M. Haftani, H. A. Chehreh, A. Mehinrad et al., "Practical investigations on use of weighted joint density to decrease the limitations of RQD measurements," Rock Mechanics and Rock Engineering, vol. 49, no. 4, pp. 1551-1558, 2015.

[49] W. Zhang, Q. Wang, J. P. Chen et al., "Determination of the optimal threshold and length measurements for RQD calculations," International Journal of Rock Mechanics and Mining Sciences, vol. 51, no. 1, pp. 1-12, 2012b.

[50] J. S. Li and S. S. Zuo, "Spatial variation of rock quality designation (RQD) in fractured rock masses," Journal of Jilin University (Earth Science Edition), vol. 44, no. 3, pp. 946-953, 2014, in Chinese.

[51] Z. Senz and A. Kazi, "Discontinuity spacing and RQD estimates from finite length scanlines," International Journal of Rock Mechanics and Mining Science \& Geomechanics Abstracts, vol. 21, no. 4, pp. 203-212, 1984.

[52] E. Hoek and E. T. Brown, "The Hoek-Brown failure criterion and GSI - 2018 edition," Journal of Rock Mechanics and Geotechnical Engineering, vol. 11, no. 3, pp. 445-463, 2019.

[53] G. X. Wang, S. F. Xiao, and J. P. Chen, "Study on application of 3d network of discontinuities to RQD," Chinese Journal of Rock Mechanics and Engineering, vol. 21, no. 12, pp. 17611764, 2002b, in Chinese. 